\title{
Non-alcoholic fatty liver disease in patients with inflammatory bowel disease might boost cardiovascular disease burden
}

\author{
Dimitrios Ioannis Patoulias
}

First Department of Internal Medicine, General Hospital “Hippokration”, Thessaloniki, Greece

Submitted: 22 August 2019

Accepted: 5 November 2019

Arch Med Sci Atheroscler Dis 2019; 4: e250-e251

DOI: https://doi.org/10.5114/amsad.2019.90147

Copyright $@ 2019$ Termedia \& Banach

Recent data suggest that the presence of inflammatory bowel disease (IBD) increases the odds for non-alcoholic fatty liver disease (NAFLD) almost by 4.5 times [1], with the latter representing the most common hepatobiliary manifestation among IBD patients [2]. Independent risk factors of hepatic steatosis in IBD patients include disease relapse rate, surgery for disease and its complications and extent of intestinal involvement [3]. However, this association also encompasses several cardio-metabolic risk factors, such as type 2 diabetes, hypertension, insulin resistance, metabolic syndrome and obesity [4, 5].

Patients with IBD face the risk of premature cardiovascular disease (CVD), with cardiovascular risk being greater for young rather than older adults [6]; a previous meta-analysis confirmed that IBD patients exhibit significantly higher carotid-femoral pulse wave velocity (cfPWV), a validated marker of aortic stiffness, compared to controls, documenting the early vascular ageing occurring during the disease course [7]. In clinical practice, it has been established that patients with IBD feature greater odds for ischemic heart disease and cerebrovascular disease, compared to controls, while younger patients are more susceptible to cerebrovascular accidents [8]. Interplay between chronic inflammation and conventional risk factors accounts for the development of CVD in this population [8]. On the other hand, it has also been established that NAFLD itself is closely related to fatal and non-fatal cardiovascular events; however, pathophysiologic causality has yet to be determined [9].

Interestingly, even underweight patients with IBD have greater liver fat content, corresponding to mild/moderate liver steatosis, compared to healthy controls [10]. Lean NAFLD patients exhibit the entire spectrum of metabolic syndrome, sharing a common metabolic profile with obese NAFLD patients [11]. Based on the fact that subclinical CVD is usually present even in lean NAFLD patients [12], it seems that IBD and NAFLD concomitance might enhance the overall cardiovascular risk.

Unfortunately, current evidence is not adequate to shed light on the underlying pathophysiologic mechanisms accounting for this close relationship between NAFLD and IBD. According to a recently published experimental study, mice fed a high-fat diet in order to develop a NAFLD phenotype exhibited significantly higher serum high mobility group box 1 (HMGB1) levels, which triggered a significant increase in the levels of monocyte chemoattractant protein-1 (MCP-1), transforming growth factor $\beta$ (TGF- $\beta$ ), interleukin-1 $\beta$ (IL-1 $\beta)$ and interferon- $\gamma$ (IFN- $\gamma)$ in mouse intesti-

\author{
Corresponding author: \\ Dr. Dimitrios loannis \\ Patoulias \\ First Department \\ of Internal Medicine \\ General Hospital \\ "Hippokration" \\ Thessaloniki, Greece \\ Phone: +30 6946900777 \\ E-mail: dipatoulias@gmail. \\ com
}


nal tissue via a NOX-2 dependent manner, finally resulting in intestinal inflammation [13]. Additionally, it was demonstrated that HMGB1-induced NADPH oxidase 2 (NOX2) activation increased expression of toll-like receptor 4 (TLR4)-pathway cytokines IL-1 $\beta$ and interleukin-6 (IL-6), crucial for the promotion of intestinal inflammation [13]. The latter cytokines have been established as cardiovascular risk markers for the last two decades $[14,15]$. However, and despite the fact that circulating HMGB1 might function as a diagnostic and prognostic biomarker of CVD [16], one could only speculate that HMGB1-induced signaling pathway is partially responsible for systemic inflammation and subsequently for the development of CVD among patients with NAFLD-IBD concomitance.

It should be highlighted that, despite the established knowledge that NAFLD is associated with increased risk for the development of CVD, two recently published large cohort studies provide contradictory results on the monitoring of these patients: one suggests that NAFLD patients should be closely monitored for CVD disease prevention [17], while the other proposes that cardiovascular risk stratification in NAFLD patients should be made in the same way as in the general population [18]. We also know that cardiovascular evaluation is rather conservative for asymptomatic NAFLD patients, based on current recommendations, unless they feature intermediate or high risk or have progressed to NASH cirrhosis [19]. However, no data exist concerning cardiovascular assessment in patients with NAFLD-IBD concomitance. As the latter might boost overall CVD risk, physicians should also consider, besides utilization of risk prediction scores and thorough laboratory investigation, performance of echocardiography, along with measurement of arterial stiffness and carotid intima media thickness, if available.

In conclusion, these patients require close monitoring and a multilevel diagnostic and therapeutic approach irrespective of body mass index, early after the onset of the disease. Further prospective studies are needed, in order to establish the interconnection between this triad at a pathophysiologic level and assess the safety and efficacy of potential, multitargeted treatment options.

\section{Conflict of interest}

The author declares no conflict of interest.

\section{References}

1. Likhitsup A, Dundulis J, Ansari S, et al. Prevalence of non-alcoholic fatty liver disease on computed tomography in patients with inflammatory bowel disease visiting an emergency department. Ann Gastroenterol 2019; 32: 1-4.

2. Silva J, Brito BS, Silva INN, et al. Frequency of hepatobiliary manifestations and concomitant liver disease in inflammatory bowel disease patients. Biomed Res Int 2019; 2019: 7604939.

3. Sartini A, Gitto S, Bianchini M, et al. Non-alcoholic fatty liver disease phenotypes in patients with inflammatory bowel disease. Cell Death Dis 2018; 9: 87.

4. Zou ZY, Shen B, Fan JG. Systematic review with metaanalysis: epidemiology of nonalcoholic fatty liver disease in patients with inflammatory bowel disease. Inflamm Bowel Dis 2019; 25: 1764-72.

5. Glassner K, Malaty HM, Abraham BP. Epidemiology and risk factors of nonalcoholic fatty liver disease among patients with inflammatory bowel disease. Inflamm Bowel Dis 2017; 23: 998-1003.

6. Singh S, Kullo IJ, Pardi DS, Loftus EV Jr. Epidemiology, risk factors and management of cardiovascular diseases in IBD. Nat Rev Gastroenterol Hepatol 2015; 12: 26-35.

7. Wu GC, Leng RX, Lu Q, Fan YG, Wang DG, Ye DQ. Subclinical atherosclerosis in patients with inflammatory bowel diseases: a systematic review and meta-analysis. Angiology 2017; 68: 447-61.

8. Singh S, Singh H, Loftus EV Jr, Pardi DS. Risk of cerebrovascular accidents and ischemic heart disease in patients with inflammatory bowel disease: a systematic review and meta-analysis. Clin Gastroenterol Hepatol 2014; 12: 382-93.

9. Targher G, Byrne CD, Lonardo A, Zoppini G, Barbui C. Non-alcoholic fatty liver disease and risk of incident cardiovascular disease: a meta-analysis. J Hepatol 2016; 65: 589-600.

10. Adams LC, Lübbe F, Bressem K, Wagner M, Hamm B, Makowski MR. Non-alcoholic fatty liver disease in underweight patients with inflammatory bowel disease: a case-control study. PLoS One 2018; 13: e0206450.

11. Sookoian S, Pirola CJ. Systematic review with meta-analysis: risk factors for non-alcoholic fatty liver disease suggest a shared altered metabolic and cardiovascular profile between lean and obese patients. Aliment Pharmacol Ther 2017; 46: 85-95.

12. Fracanzani AL, Petta S, Lombardi R, et al. Liver and cardiovascular damage in patients with lean nonalcoholic fatty liver disease, and association with visceral obesity. Clin Gastroenterol Hepatol 2017; 15: 1604-11.e1.

13. Chandrashekaran V, Seth RK, Dattaroy D, et al. HMGB1RAGE pathway drives peroxynitrite signaling-induced IBD-like inflammation in murine nonalcoholic fatty liver disease. Redox Biol 2017; 13: 8-19.

14. Ridker PM, Hennekens CH, Buring JE, Rifai N. C-reactive protein and other markers of inflammation in the prediction of cardiovascular disease in women. $\mathrm{N}$ Engl J Med 2000; 342: 836-43.

15. Ridker PM, Rifai N, Stampfer MJ, Hennekens CH. Plasma concentration of interleukin- 6 and the risk of future myocardial infarction among apparently healthy men. Circulation 2000; 101: 1767-72.

16. Raucci A, Di Maggio S, Scavello F, D’Ambrosio A, Bianchi ME, Capogrossi MC. The Janus face of HMGB1 in heart disease: a necessary update. Cell Mol Life Sci 2019; 76: 211-29.

17. Sinn DH, Kang D, Chang Y, et al. Nonalcoholic fatty liver disease and the incidence of myocardial infarction: a cohort study. J Gastroenterol Hepatol 2019 Sep 11. doi: 10.1111/jgh.14856. [Epub ahead of print].

18. Alexander M, Loomis AK, van der Lei J, et al. Non-alcoholic fatty liver disease and risk of incident acute myocardial infarction and stroke: findings from matched cohort study of 18 million European adults. BMJ 2019; 367: 15367.

19. Choudhary NS, Duseja A. Screening of cardiovascular disease in nonalcoholic fatty liver disease: whom and how? J Clin Exp Hepatol 2019; 9: 506-514. 